\title{
Association of Peripheral Blood RASSF1A and CDKN2A Methylation Status with Smoking Behaviour in Nasopharyngeal Carcinoma
}

\author{
Erika Diana Risanti ${ }^{1,2}$, Aditya Kurniawan², Laila Wahyuningsih ${ }^{3}$, Ery Kus Dwianingsih $^{3}$, \\ Hanggoro Tri Rinonce ${ }^{3}$, Jajah Fachiroh ${ }^{4, *}$ \\ ${ }^{1}$ Faculty of Medicine, Muhammadiyah University of Surakarta, Jl. A. Yani, Pabelan, Surakarta, Indonesia \\ ${ }^{2}$ Postgraduate Study on Biomedical Science, Faculty of Medicine Universitas Gadjah Mada, Jl. Farmako Sekip Utara, Yogyakarta, Indonesia \\ ${ }^{3}$ Department of Anatomical Pathology, Faculty of Medicine, Universitas Gadjah Mada, Jl. Farmako Sekip Utara, Yogyakarta, Indonesia \\ ${ }^{4}$ Department of Histology and Cell Biology, Faculty of Medicine, Universitas Gadjah Mada, Jl. Farmako Sekip Utara, Yogyakarta, Indonesia \\ *Corresponding author. E-mail: jajahfachiroh@ugm.ac.id
}

Received date: Sep 29, 2017; Revised date: Dec 3, 2017; Accepted date: Jan 4, 2018

\section{Abstract}

ACKGROUND: Hypermethylation of RASSF1A and $C D K N 2 A$ is one of epigenetic factor underlies nasopharyngeal carcinoma (NPC) development. Smoking behavior as an NPC's risk factor causes aberrant DNA methylation. RASSF1A and CDKN2A promoter hypermethylation from peripheral blood cells correlates with smoking behavior. The use of body fluids including peripheral blood as a specimen for DNA methylation analyzes are widely developed, as less invasive method compared to the use of tissue biopsy. This study aims to observe the association between RASSF1A and CDKN2A methylation in peripheral blood and smoking behavior among NPC patients.

METHODS: Newly diagnosed NPC subjects were recruited from ear-nose-throat (ENT) outpatient clinic of Dr. Sardjito Hospital, Yogyakarta. DNA from buffycoat of 19 smokers and 20 non-smokers NPC's patients were isolated. Bisulphite modification was applied to $500 \mathrm{ng}$ of the isolated DNA. The methylation status was detected by MSP (methylation-specific polymerase chain reaction (PCR)). The association between smoking status and promoter hypermethylation was analysis using Chi-Square test.

RESULTS: MSP analysis of RASSF1A showed that $68.42 \%$ smoker and 75\% non-smoker NPC's patients were methylated. MSP analysis of CDKN2A showed that $21.05 \%$ smoker and 25\% non-smoker NPC's patients were methylated. There was no association between smoking behavior with RASSF1A and CDKN2A methylation $(p>0.05)$.

CONCLUSION: Statistical analysis showed that smoking behavior is not associated with methylation of RASSF $1 A$ and $C D K N 2 A$ among NPC's patients.

KEYWORDS: DNA methylation, CDKN2A, RASSF1A, Nasopharyngeal carcinoma, Smoking

Indones Biomed J. 2018; 10(2): 123-7

\section{Introduction}

Nasopharyngeal carcinoma (NPC) is a rare malignancy worldwide, with incidence of less than $1 / 100.000$ populations.(1) However, in some regions, including South-east Asia, the incidence is high. The incidence of NPC in Indonesia is predicted as high as 5-7/100.000 populations.(2) Late clinical presentation and bad prognosis are characteristic of NPC. $(3,4)$ Cigarette smoking is common risk of cancer, including nasopharyngeal carcinoma. Carcinogenic materials in cigarette such as arsenic, chromium, formaldehyde, polycrilic acromatic hydrocarbons and nitrosamines causes DNA damage. Cigarette smoke is considered one of the most powerful environmental modifiers of DNA methylation. The specific mechanisms 
of how cigarette smoke may alter DNA methylation are becoming better understood.(5) Cigarette smoking causes DNA damage, hypoxia, activated DNA-binding protein and accumulation of DNA methyltransferase. This condition can cause an alteration of DNA methylation.

Hypermethylation DNA is the most studied epigenetic alteration in cancer. It has been described for variety of cancer including NPC. Hypermetilation DNA of promoter tumor suppressor gene causes expression silences from the gene. These genes are very important for controlling proliferation, cell cycle, cell motility and apoptosis. Aberration of DNA methylation mostly caused by environmental factors. $(6,7)$ RASSF1A and CDKN2A genes are tumor supressor genes that involved in NPC pathogenesis. Several studies were demonstrated that both genes has been methylated in NPC. (8-10) Cigarette smoking was associated with RASSF $1 A$ and $C D K N 2 A$ methylation status among lung cancer patients. (11) However, there has been no study that correlates smoking behavior and RASSF1A and CDKN2A methylation status in NPC.

Methylation study in NPC using leukocytes has never been done. However, there was a study in RASSF1A and CDKN2A methylation using leukocytes. This study was consist of smoker and non-smoker groups and the results showed that RASFF1A and p16 (CDKN2A) methylation were higher in smokers than non-smokers group.(20)

The use of body fluids including peripheral blood as a specimen for DNA methylation analyzes are widely developed. That specimens were obtained less invasively compared to tissue biopsy, and might be resampled more frequently. Detection of DNA methylation in peripheral blood have been reported in several studies, including lung cancer (12), pancraetic cancer (13) and ovarium cancer (14). This study reports the observation of RASSF1A and $C D K N 2 A$ methylation in peripheral blood, and their association with smoking behavior among NPC patients.

\section{Methods}

This study used newly diagnosed NPC subjects were recruited from ear-nose-throat (ENT) outpatient clinic Dr. Sardjito General Hospital, Yogyakarta, as part of NPC Hospital-based case-control study (2012-2016; Principle investigator: Jajah Fachiroh). Smoking status was determined through questionnaire. Smokers are those who smoke regularly for at least a year, while non-smokers are those who never smoke regularly for at least one year in their life. Ethical permission was given by Faculty of Medicine
UGM Internal review board number KE/FK/730/EC, and informed consent was given prior to data collection.

We selected 19 NPC who smoked and 20 NPC who did not smoke to enter this study. All subjects were male and Javanish. DNA from buffycoat of peripheral blood was extracted by using Geneaid DNA Isolation Kit (Geneaid Biotech. Ltd, Taiwan). Five-hundred ng of DNA was used for bisulfite treatment using commercially kit Cells-to-CpG Bisulfite Modification (Applied Biosystem, Massachusetts, USA).

MSP (Methylation-specific polymerase chain reaction (PCR)) was used as methylation analysis. The PCR specific primer consist of methylation and unmethylation sequences. This four pairs of specific primer was obtained from previous study. $(15,16)$ Modified DNA from breast tissue which known RASSF1A and CDKN2A metylation status was used as positive control. Distilled water was used for negative control. Platinum ${ }^{\circledR}$ Taq Polymerase (Invitrogen, Massachusetts, USA) was used for amplification. Fluorosafe DNA stain (1st Base, Singapore) was used for nucleic acid stain. Electroforesis's product was visualized using UV translumination.

Association between smoker and non-smoker NPC's patients was analysis using Chi-Square. Two sided $p \leq 0.05$ was considered as statistically significant. All statistical calculations was performed using SPSS ver. 23 (SPSS Inc., Chicago, USA).

\section{Results}

Subjects characteristic were divided based in their smoking status (Figure 1). Analysis showed that there were no differences in age group and histopathological characteristics. Median age group and years of smoking was grouped on the median value in smoker NPC's patients and non-smoker NPC's patients. WHO histopathological type in both groups was not significantly different. Smoking duration ranged from 8-61 years with median of years 32 . Number of cigarette smoked was ranged from 2-36 cigarettes per day with median 10 cigarette per day (Table 1).

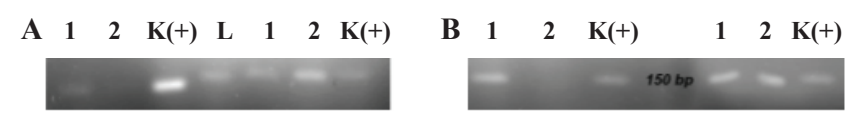

Figure 1. MSP result for RASSF1A (A) and CDKN2A (B) methylation detection. (A) Sample 1 is a methylation sample and sample 2 is an unmethylation sample. (B) Sample 1 is a methylation sample and sample 2 is an unmethylation sample. 
Table 1. Subject Characteristics. There were no significance differences in age group between smoker and non-smoker NPC's patients. There were no significant differences in WHO type of cancer between smokers and non-smokers subjects.

\begin{tabular}{lccc}
\hline \multicolumn{1}{c}{ Subject Characteristics } & $\begin{array}{c}\text { Case (\%) } \\
\mathbf{n}=\mathbf{1 9}\end{array}$ & $\begin{array}{c}\text { Control (\%) } \\
\mathbf{n}=\mathbf{2 0}\end{array}$ & $\boldsymbol{p}^{*}$ \\
\hline Age (years) & & & \\
$\quad$ Mean $( \pm$ SD) & $50.90( \pm 10.21)$ & $51.15( \pm 11.18)$ & 0.444 \\
$\quad$ Median & 51.5 & 53.5 & \\
$\quad$ Range & $33-76$ & $25-66$ & \\
\hline WHO Type & & & \\
$\quad$ Type 1 & $0(0)$ & $0(0)$ & 1000 \\
$\quad$ Type 2 & $0(0)$ & $1(5)$ & \\
$\quad$ Type 3 & $19(100)$ & $19(95)$ & \\
\hline Smoking duration (years) & & & \\
$\quad<\mathbf{3 2}$ & $9(47.4)$ & & \\
$\geq \mathbf{3 2}$ & $10(52.6)$ & \\
$\quad$ Range & $8-61$ & \\
\hline Cigarette per day & & \\
$\quad<\mathbf{1 0}$ & $6(31,6)$ & \\
$\geq \mathbf{1 0}$ & $13(68.4)$ & \\
$\quad$ Range & $2-36$ &
\end{tabular}

DNA methylation analysis used MSP. MSP methylation products of RASSF1A is $94 \mathrm{bp}$ meanwhile unmethylation products is $105 \mathrm{bp}$. Methylation products of $C D K N 2 A$ is $150 \mathrm{bp}$ and unmethylation products is $151 \mathrm{bp}$ (Figure 1). Meanwhile the primer specific for MSP in both genes are shown in Table 2.

MSP analysis of RASSF $1 A$ showed that $68.42 \%$ smoker and $75 \%$ non-smoker NPC's patients were methylated. MSP analysis of $C D K N 2 A$ have a similar result. It showed that $21.05 \%$ smoker and $25 \%$ non-smoker NPC's patients were methylated (Table 3).

Smoking behaviour of NPC's patients was not significantly associated with RASSF1A and CDKN $2 A$ methylation. This results was proved using Chi-Square test with $p$ value $>0.05$ (Table 3 ).

\section{Discussion}

Aberrant cell growth is commonly reported as result of DNA mutation. However, compared to other type of cancers, methylation is more often reported to occur in NPC.(7) Gene methylation occurs in the tissue of nasopharynx or Epstein-Barr virus (EBV) gene to support its oncogenic role.(4) Several studies reported the occurrence of promoter methylation in tumor suppressor genes in NPC.(8-10) All of these studies used tissue from the nasopharynx as reflecting in situ epigenetic changes.

However, the use of nasopharynx tissue has been limited by its quantity and quality. Nasopharyngeal carcinoma is not an operable cancer, biopsy yielding small cut of tissue, that used mostly for histopathological diagnosis preparation (formalin-fixed paraffin embedded (FFPE)), biopsy is an invasive method, operated only by clinical specialist; thus, limit the frequent use. The use of nasopharyngeal brush which targets the exfoliating epithelial cells was also suggested.(8) This technique

Table 2. Primer specific for MSP in both genes.

\begin{tabular}{cccc}
\hline Gene & Primer & \multicolumn{1}{c}{ Sequences } & Product \\
\hline RASSF1A & Methylation & F:5'-GTGTTAACGCGTTGCGTATC-3' & $94 \mathrm{bp}$ \\
& & R:5'-AACCCCGCGAACTAAAAACGA-3' & \\
\cline { 2 - 4 } & Unmethylation & F:5'-TTTGGTTGGAGTGTGTTAATGTG-3' & $105 \mathrm{bp}$ \\
& & R:5'-CAAACCCCACAAACTAAAAACAA-3' & \\
\hline CDKN2A & Methylation & F:5'-TTATTAGAGGGTGGGGCGGATCGC-3' & $150 \mathrm{bp}$ \\
& & R:5'-GACCCCGAACCGCGACCGTAA-3' & \\
\cline { 2 - 4 } & Unmethylation & F: 5'-TTATTAGAGGGTGGGGTGGATTGT-3' & $151 \mathrm{bp}$ \\
& & R:5'-CAACCCCAAACCACAACCATAA-3' & \\
\hline
\end{tabular}

Table 3. The association between smoking behaviour and RASSF1A and CDKN2A methylation.

\begin{tabular}{llccccc}
\hline & & Methylation & $\mathbf{\%}$ & Unmethylation & $\mathbf{\%}$ & $\boldsymbol{p}^{*}$ \\
\hline RASSF1A & Smoker & 13 & 68.42 & 6 & 31.58 & 0.731 \\
& Non-smoker & 15 & 75 & 5 & 25 & \\
\hline \multirow{2}{*}{ *DKN2A } & Smoker & 4 & 21.05 & 15 & 78.95 & 1 \\
& Non-smoker & 5 & 25 & 15 & 75 & \\
\hline
\end{tabular}


required ENT specialist to handle guided endoscopy to target the sampling area.

The use of peripheral blood is considered as a better replacement for less invasiveness and capacity for more frequent sampling, therefore suitable to be used for screening or follow up. Cell-free DNA (cfDNA) has recently being used to replace tissue, with the assumption that DNA was originated from cancer tissues (6) as the result of apoptosis. However, the efficiency of cfDNA is quite low. On the other hand, the use of whole blood/ blood cells is also an option. Immunologic process related to inflammation in cancer development has been suggested to lead the changes in the leucocyte populations. That changes could alter epigenetic in peripheral blood.(17) Development of DNA methylation study in peripheral blood is expected can describe tissue's methylation condition. The methylation study in brain tissue, breast cancer and colorectal cancer showed that peripheral blood's methylation couldn't describe condition in tissues.(18,19) However, the study in lung tissue showed different result.(12) Within limited sample we could demonstrate parallel detection of RASF1A in FFPE tissue of nasopharynx and blood cells (data not shown).

Tobacco smoking has been reported to cause epigenetic changes, as reported by Huang, et al.(11) Within this study, we found no association between smoking behavior and RASSF $1 A$ and CDKN2A methylation in NPC's patients. In both smokers and non-smokers we found similar distribution of RASSF $1 A$ and CDKN2A methylation. Previous study using FFPE of the nasopharynx and exfoliate of nasopharynx tissue showed that that RASSF1A and $C D K N 2 A$ methylation status in NPC's patients were higher than non-cancer patients. $(8,10)$ Study done by Deep, et al., showed that methylation in both genes were high among smokers compared to non-smokers. $(12,20)$ Which shed light for the possibility to use these process as biomarkers for smoking. In our hand, we could not confirm the difference of smoking behavior, instead to confirm parallel results of RASSF1A and CDKN2A methylations in NPC. This study has limitation, including the absence of passive smoking status, and limitation of sample numbers.

\section{Conclusion}

As a conclusion, within this study we were able to detect DNA methylation of RASSF1A and CDKN2A in the cells of the peripheral blood. DNA methylation of RASSF $1 A$ and $C D K N 2 A$ in peripheral blood were not associated with smoking behavior among NPC subjects. Further, analysis by using healthy blood donor may help us to conclude the status of methylation of RASSF1A and CDKN2A, either as marker for smoking or for cancer.

\section{Acknowledgment}

We gratefully acknowledge Ministry of Research, technology, and higher education for PUPT 2016 (Principle investigator: Jajah Fachiroh), and Dana Masyarakat UGM 2016 (Principle investigator: Hanggoro Tri Rinonce) for grant fundings. We also would like to thank Saihas Suhda for providing buffycoat DNA samples, and Sri Fatmawati for technical assistance.

\section{References}

1. Tsao SW, Yip YL, Tsang CM, Pang PS, Lau VMY, Zhang G, et al. Etiological factors of nasopharyngeal carcinoma. Oral Oncol. 2014; 50: 330-8.

2. Adham M, Middeldorp JM, Kurniawan AN, Muhtadi AI, Roezin A, Hermani B, et al. Nasopharyngeal carcinoma in Indonesia: epidemiology, incidence, signs, and symptoms at presentation. Chin J Cancer. 2013; 31: 185-96.

3. Thompson LDR. Update on Nasopharyngeal Carcinoma. Head Neck Pathol. 2007; 1: 81-6.

4. Chou J, Ling YC, Kim J, You L, Xu Z, He B, et al. Nasopharyngeal carcinoma review of molecular mechanisms of tumorigenesis. Head Neck Cancer. 2008; 30: 946-63.

5. Lee KWK, Pausova Z. Cigarette smoking and DNA methylation. Front Genet. 2013; 4: 132. doi: 10.3389/fgene.2013.00132.

6. Yokoi K, Yamashita K, Watanabe M. Analysis of DNA methylation status in bodily fluids for early detection of cancer. Int J Mol Sci. 2017; 18: E735. doi: 10.3390/ijms18040735.

7. Dai W, Zheng H, Cheung AKL, Lung ML. Genetic and epigenetic landscape of nasopharyngeal carcinoma. Chinese Clin Oncol. 2016; 5: 16. doi: $10.21037 / \mathrm{cco} .2016 .03 .06$.

8. Hutajulu SH, Indrasari SR, Indrawati LPL, Harijadi A, Duin S, Haryana SM, et al. Epigenetic markers for early detection of nasopharyngeal carcinoma in a high risk population. Mol Cancer. 2011; 10: 48. doi: 10.1186/1476-4598-10-48.

9. Tian F, Yip SP, Kwong DLW, Lin Z, Yang Z, Wu VWC. Promoter hypermethylation of tumor suppressor genes in serum as potential biomarker for the diagnosis of nasopharyngeal carcinoma. Cancer Epidemiol. 2013; 37: 708-13.

10. Challouf S, Ziadi S, Zaghdoudi R, Ksiaa F, Ben Gacem R, Trimeche M. Patterns of aberrant DNA hypermethylation in nasopharyngeal carcinoma in Tunisian patients. Clin Chim Acta. 2012; 413: 795802.

11. Huang $T$, Chen $X$, Hong Q, Deng Z, Ma H, Xin Y, et al. Metaanalyses of gene methylation and smoking behavior in non-small cell lung cancer patients. Sci Rep. 2015; 5: 8897. doi: 10.1038/ srep08897 
12. Woodson K, Mason J, Choi S, Woodson K, Mason J, Choi S, et al. Hypomethylation of p53 in peripheral blood DNA is associated with the development of lung cancer hypomethylation of p53 in peripheral blood DNA is associated with the development of lung cancer. 2001; 10: 69-74.

13. Pedersen KS, Bamlet WR, Oberg AL, de Andrade M, Matsumoto ME, Tang H, et al. Leukocyte DNA methylation signature differentiates pancreatic cancer patients from healthy controls. PLoS One. 2011; 6: 1-9. doi: 10.1371/journal.pone.0018223.

14. Teschendorff AE, Menon U, Gentry-Maharaj A, Ramus SJ, Gayther $\mathrm{SA}$, Apostolidou S, et al. An epigenetic signature in peripheral blood predicts active ovarian cancer. PLoS One. 2009; 4: e8274. doi: 10.1371/journal.pone.0008274.

15. Herman JG, Graff JR, Myohanen S, Nelkin BD, Baylin SB. Methylation-specific PCR: a novel PCR assay for methylation status of CpG islands. Proc Natl Acad Sci. 1996; 93: 9821-6.

16. Lo K, Kwong J, Hui AB, Chan SY, To K, Chan AS, et al. High frequency of promoter hypermethylation of RASSF1A in nasopharyngeal carcinoma. Cancer Res. 2001; 61: 3877-81.

17. Li L, Choi JY, Lee KM, Sung H, Park SK, Oze I, et al. DNA methylation in peripheral blood: a potential biomarker for cancer molecular epidemiology. J Epidemiol. 2012; 22: 384-94.

18. Walton E, Hass J, Liu J, Roffman JL, Bernardoni F, Roessner V, et al. Correspondence of DNA methylation between blood and brain tissue and its application to schizophrenia research. Schizophr Bull. 2016; 42: 406-14.

19. Li X, Wang Y, Zhang Z, Yao X, Ge J, Zhao Y. Correlation of MLH1 and MGMT methylation levels between peripheral blood leukocytes and colorectal tissue DNA samples in colorectal cancer patients. Oncol Lett. 2013; 6: 1370-6.

20. Deep JS, Sidhu S, Chandel A, Thapliyal S, Garg C. Aberrant methylation in promoters of GSTP1, p16, p14, and RASSF1A genes in smokers of North India. ISRN Pulmonol. 2012; 2012: 247631. doi: $10.5402 / 2012 / 247631$ 